\title{
Randomization with asymmetric information
}

\author{
Richard Arnott* \\ and \\ Joseph E. Stiglitz**
}

It is by now well known that, in the presence of moral hazard or adverse selection, randomization of insurance premiums and benefits may be Pareto efficient. This article provides a typology of the various forms that randomization may take, derives necessary or sufficient conditions for the desirability of these various forms of randomization, obtains some simple characterization theorems of the efficient random policies, gives some intuition behind the results, and considers why randomization appears to occur less often in practice than the theory suggests it should.

\section{Introduction}

During the past fifteen years, an extensive literature has developed analyzing the structure of adverse-selection and moral-hazard (principal - agent) problems. Early on it was recognized that these problems may not have the usual convexity-concavity properties so familiar to economists; and only a few years later, it was realized that this in turn implies that in a variety of circumstances randomization is efficient. ${ }^{1}$

The aims of this article are to provide a typology of the various forms that randomization may take, to provide necessary or sufficient conditions for the desirability ${ }^{2}$ of these various forms of randomization in the context of the standard models of an insurance market with moral hazard or adverse selection, to provide some simple characterization theorems of the efficient random policies, to provide some intuition behind the results, and to consider why randomization appears to occur less often in practice than the theory suggests it should.

Many of our results are new. Some (Propositions 2, 4, and 6), however, are not. In

* Boston College and Queens University.

** Stanford University.

This article was written in conjunction with the authors' research project on moral hazard, financial support for which has been provided by the National Science Foundation, the Social Sciences and Humanities Research Council of Canada, and the Olin Foundation. Part of the work on the article was done when Stiglitz was a Visiting Scholar at the Hoover Institution, Stanford. We are grateful to the referees and the editors, especially Ken Judd, whose comments resulted in substantial expansion and revision which have, we hope, improved the clarity of the article. Any remaining errors are our responsibility.

${ }^{1}$ The earliest studies were in the context of the problems of optimal taxation (Weiss, 1976; Stiglitz, 1982a, 1982b; Balcer and Sadka, 1982).

${ }^{2}$ When we say that randomization is "desirable," we mean that there exists a Pareto-improving randomization. Also, if randomization is desirable, it will be used by insurance firms in competitive equilibrium (as long as they can observe their clients' total purchases of insurance) and by a monopoly insurer. 
particular, several recent articles have investigated the desirability of randomization of insurance contracts in the presence of moral hazard (Holmström, 1979; Gjesdal, 1982; Fellingham, Kwon, and Newman, 1984). All treat a continuum of possible outcomes, which tends to obscure intuition. We rederive their results for the two-outcome case by using derivations that highlight the intuition.

Section 2 treats randomization with moral hazard, and Section 3 treats randomization with adverse selection. The concluding section contains a discussion of why randomization is not so common as theory suggests it should be, which casts doubt on the appropriateness of several of the standard assumptions employed in the incentives and contract literatures.

Throughout the moral-hazard section we cast the problem in terms of a monopoly insurer who maximizes profits subject to providing his clients with an exogenously specified level of expected utility and subject also to the relevant incentive-compatibility constraints. This allows us to characterize Pareto-efficient contracts. In the adverse-selection section, meanwhile, we found it more natural to develop the analysis in the context of a competitive insurance market. Throughout the article competitive equilibrium (when it exists) corresponds to the solution of the monopoly problem with expected utility at the competitive equilibrium level(s). ${ }^{3}$ Thus, when we say that randomization is desirable, we mean that randomization is Pareto efficient, is profitable for a monopoly insurer, and is a characteristic of competitive equilibrium.

\section{Moral hazard}

- Consider the simplest moral-hazard setting. There are a large number of identical individuals, each of whom realizes one of two possible outcomes or events. Either an accident does not occur, in which case the individual receives $w$ before insurance, or an accident ${ }^{4}$ does occur, in which case the individual receives $w-d$, where $d$ is the accident damage. The probability that an accident occurs depends on the individual's accident-prevention effort, $e$; i.e., $p=p(e)$. We assume that $p^{\prime}<0$ and $p^{\prime \prime}>0$, and that the individual always expends some effort in the monopoly solution. Also, individuals' accident probabilities are statistically independent.

Moral hazard arises because an insurer is unable to observe effort. As a result, insurance contracts cannot be written contingent on effort. We assume that the insurer can, however, observe his clients' total insurance purchases. ${ }^{5}$ In these circumstances a nonrandom insurance contract will specify a (net) insurance payout or benefit $\alpha$ payable to an individual if an accident occurs and an insurance premium $\beta$ payable by the individual if an accident does not occur. Thus, consumption $(y)$ is $w-d+\alpha$ when an accident does occur, and $w-\beta$ when it does not.

The individual's expected utility is

$$
E U=(1-p(e)) U_{0}(w-\beta, e)+p(e) U_{1}(w-d+\alpha, e),
$$

where $U_{0}$ is the no-accident utility function and $U_{1}$ the accident utility function. We assume that

$$
\frac{\partial U_{j}}{\partial y}>0, \quad \frac{\partial^{2} U_{j}}{\partial y^{2}}<0, \quad \frac{\partial U_{j}}{\partial e}<0 \quad \text { for } \quad j=0,1 .
$$

Note that this specification permits utility functions to be outcome-contingent; the accident may have nonpecuniary effects or influence tastes. We shall have occasion to particularize (1):

\footnotetext{
${ }^{3}$ This equivalence can be established on the basis of arguments presented in Arnott and Stiglitz [1988b].

4 "Accident" may be reinterpreted as large damage conditional on an accident's occurring, and "no accident" as small damage conditional on an accident's occurring.

${ }^{5}$ The assumption of a monopoly insurer implies this assumption. This assumption is, however, consequential in the competitive equilibrium interpretation (Arnott and Stiglitz, 1988b).
} 
(i) separable utility function

$$
E U=(1-p(e)) u_{0}(w-\beta)+p(e) u_{1}(w-d+\alpha)-e
$$

with $u_{j}^{\prime}>0$ and $u_{j}^{\prime \prime}<0$ for $j=0,1$, and effort is measured in terms of the disutility it causes;

(ii) separable, event-independent utility function

$$
E U=(1-p(e)) u(w-\beta)+p(e) u(w-d+\alpha)-e .
$$

The individual chooses effort to maximize expected utility, while taking the parameters of the insurance contract as given. This yields a function or correspondence relating effort to the parameters of the insurance contract, $e=e(\alpha, \beta)$, which, when substituted into the expected utility function, $E U$, yields expected utility as a function of $\alpha$ and $\beta, v(\alpha, \beta)$. When constrained to choosing a nonrandom contract, the insurer's problem is to choose $(\alpha, \beta)$ to maximize profits subject to providing his clients with a given level of expected utility. ${ }^{6}$

The question to be addressed is: under what circumstances does randomization of the parameters of the insurance contract result in a welfare improvement?

The basic intuition behind why randomization might be desirable is this. Moral hazard causes a tradeoff between risk-bearing and incentives, since if full insurance were provided, the insured would take little or no accident-prevention effort. As a result, the deterministic monopoly insurance contract typically entails the insured's receiving less than full insurance and expending less than the first-best (i.e., with effort observable) level of effort. A compensated randomization of insurance may cause the insured to increase effort by enough that profits are increased. The techniques of Rothschild and Stiglitz (1970) can be used to ascertain conditions under which a mean-preserving randomization of, say, the payout will increase effort at accident avoidance; randomization will do so provided only that the firstorder condition for effort is convex in the payout. Randomization also has a direct, negative effect on risk-averse individuals. Thus, whether randomization is desirable depends on a careful balancing of the welfare gains from the mitigation of the moral-hazard problem with the welfare losses from increased risk. While the latter depend on the degree of risk aversion (which depends on first and second derivatives of the utility function), the former depend on the degree of concavity of the first-order condition for effort (which depends on first, second, and third derivatives of the utility function). There appears to be no a priori reason why the effort effect should not outweigh the risk effect. This intuition turns out to be correct. $^{7}$

The forms of randomization. We shall consider two types of randomization. If the realization of the random policy occurs before the insured individual makes his effort decision, we shall say that the randomization is ex ante; if it occurs after his effort decision, ex post. In all cases, we assume that the individual knows fully the nature of the randomization being undertaken. With ex post randomization, the insurer quotes a set of random policies $\left\{\left(\alpha_{i}, \beta_{i}, Q_{i}\right) \mid i=1, \ldots, n\right\}$, which we term the insurance contract, where $Q_{i}\left(\sum_{i} Q_{i}=1\right)$ is

the probability that policy $i$ will be assigned to the individual after he has made his effort decision. The individual makes his effort decision and only afterwards finds out which of the policies the insurance company has assigned him. With ex ante randomization, the insurer again quotes a set of random policies $\left\{\left(\alpha_{i}, \beta_{i}, Q_{i}\right) \mid i=1, \ldots, n\right\}$ but now $Q_{i}$ is the probability that policy $i$ will be assigned to the individual before he makes his effort decision.

\footnotetext{
${ }^{6}$ This problem is treated exhaustively in Arnott and Stiglitz (1988a).

${ }^{7}$ This intuition applies to what we shall identify in the next subsection as ex post randomization. A rather different set of arguments is relevant for ex ante randomization, as we shall show below.
} 
The individual is randomly assigned a policy and then makes his effort decision. To simplify the analysis we shall consider each form of randomization in isolation. Thus, in the subsection on ex post randomization, we exclude ex ante randomization by assumption, and in the section on ex ante randomization, we exclude ex post randomization by assumption.

Ex post randomization. The insurer chooses that insurance contract that maximizes his expected profits, subject to providing his clients with a given level of expected utility and while taking into account the dependence of effort on the terms of the contract. We can formalize the problem as a two-stage (indirect control) problem. In the first stage the individual chooses effort, while taking the parameters of the insurance contract as given, to maximize his expected utility:

$$
E U \equiv \sum_{i} \nu\left(\alpha_{i}, \beta_{i}, e\right) Q_{i}
$$

where

$$
\nu\left(\alpha_{i}, \beta_{i}, e\right) \equiv U_{0}\left(w-\beta_{i}, e\right)(1-p(e))+U_{1}\left(w-d+\alpha_{i}, e\right) p(e) .
$$

This defines

$$
\tilde{e} \equiv \underset{e}{\operatorname{argmax}} E U
$$

In the second stage the insurer maximizes his expected profit, while taking the dependence of the accident probability on effort, and the dependence of effort on the parameters of the insurance contracts, into account. In analyzing the second problem, it turns out to be convenient to embed in it the maximization condition for the first stage; we thus write the insurer's problem as

$$
\max _{\left.\left\{\left(\alpha_{i}, \beta_{i}, Q_{i}\right) \mid i=1, \ldots, n\right)\right\}}\left(\sum_{i} \beta_{i} Q_{i}\right)(1-p(\tilde{e}))-\left(\sum_{i} \alpha_{i} Q_{i}\right) p(\tilde{e})
$$

subject to

$$
\begin{gathered}
\sum_{i} \nu\left(\alpha_{i}, \beta_{i}, \tilde{e}\right) Q_{i} \geq \bar{U} \quad(\text { expected utility constraint }), \\
\sum_{i} Q_{i}=1, \\
Q_{i} \geq 0 \quad \forall i, \quad \text { and } \\
\tilde{e}=\underset{e}{\operatorname{argmax}} E U .
\end{gathered}
$$

Before proceeding to analyze (4), we state the following.

Lemma 1. In the general concave programming problem

$$
\begin{aligned}
& \max _{\left\{x_{i}, Q_{i}\right\}} \sum_{i} F\left(x_{i}\right) Q_{i} \quad \text { subject to } \quad \sum_{i} g^{j}\left(x_{i}\right) Q_{i} \leq c^{j}, \quad \sum_{i} Q_{i}=1 \\
& Q_{i} \geq 0 \quad \forall i, \quad x_{i} \geq 0 \quad \forall i,
\end{aligned}
$$

where $x_{i}$ is a vector of control variables, applied with probability $Q_{i}, j$ indexes constraints, $F(\cdot)$ is a weakly concave function and $g^{j}(\cdot)$ are strictly convex constraint functions, randomization of $x$ is undesirable.

Lemma 1 is well known and implies in this context that randomization is undesirable when the insurer's profit-maximization problem is concave.

Since (4) is not in general a concave programming problem, it appears that randomization may in some circumstances be desirable. Characterization of the solution to (4) is difficult. Two results may, however, be obtained. 
Proposition 1. With ex post randomization at most three insurance policies are needed to achieve the optimum.

Proposition 2. With separable utility functions ex post randomization is never desirable. ${ }^{8}$

The first proposition is established as follows. The first-order condition of the individual's effort choice problem satisfies

$$
\sum_{i} \frac{\partial \nu\left(\alpha_{i}, \beta_{i}, \tilde{e}\right)}{\partial e} Q_{i}=0
$$

(Because expected utility is not necessarily a concave function of effort, (5) is only a necessary condition; constraint (4d) is the necessary and sufficient condition.) One may imagine that the insurer's problem has been completely solved, and that one is told what the profitmaximizing random policies are and what the individual's level of effort is, but not the probabilities with which the optimal random policies are assigned. The choice of the $\left\{Q_{i}\right\}$ is then a standard linear programming problem with three constraints-constraints (4a) and (4b) and (5)-in addition to the nonnegativity constraints, which is solved with at most three nonzero $Q_{i}$.

Proposition 2 is proved by writing the insurer's maximization problem in such a way that Lemma 1 may be applied. Suppose that the level of effort chosen by individuals in the solution to the monopoly insurer's problem, $e^{*}$, has been determined. And let $\bar{u}_{0}$ denote the probability-weighted average of $u_{0}$ (recall (1a)) across the random contracts, i.e., $\bar{u}_{0} \equiv \sum_{i} u_{0}\left(w-\beta_{i}\right) Q_{i}$, and define $\bar{u}_{1}$ accordingly. Then the analog to constraint (4a), written in standard $\leq$ form, is

$$
-\bar{u}_{0}\left(1-p\left(e^{*}\right)\right)-\bar{u}_{1} p\left(e^{*}\right)+e^{*} \leq-\bar{U}
$$

and the analog to constraint $(4 \mathrm{~d})$ is

$$
\left(\bar{u}_{1}-\bar{u}_{0}\right) p^{\prime}\left(e^{*}\right)-1=0
$$

(since with separable utility, expected utility is a concave function of effort).

Substitute (6b) into (6a) to obtain

$$
-\bar{u}_{0}-\frac{p\left(e^{*}\right)}{p^{\prime}\left(e^{*}\right)}+e^{*} \leq-\bar{U} .
$$

Since constraints (6a) and (7a) together are equivalent to constraints (6a) and (6b) together, the insurer's problem may be rewritten as

$$
\max _{\left\{\alpha_{i}, \beta_{i}, Q_{i}\right\}}\left(\sum_{i} \beta_{i} Q_{i}\right)\left(1-p\left(e^{*}\right)\right)-\left(\sum_{i} \alpha_{i} Q_{i}\right) p\left(e^{*}\right)
$$

subject to (6a), $\sum_{i} Q_{i}=1, Q_{i} \geq 0 \forall i$, and (7a). Since this programming problem has a linear objective function and constraint functions that are strictly convex in $\left\{\alpha_{i}, \beta_{i}\right\}$, Lemma 1 applies, and so randomization is undesirable.

The "tricks" in the proof were to hold $e$ fixed, and to rewrite constraints (6a) and (6b), where $(6 \mathrm{~b})$ is not a convex constraint, as $(6 a)$ and $(7 a)$, both of which are convex constraints.

Proposition 2 and its proof are useful because they provide the basis of a general theorem characterizing sufficient conditions for randomization to be undesirable. If the utility function is not separable, the equation analogous to $(7 a)$ is

\footnotetext{
${ }^{8}$ This proposition has been proved previously for the continuum of outcomes case by Holmström (1979). Our proof, however, is new.
} 


$$
\varphi\left(y_{0}, y_{1}, e^{*}\right) \equiv-\bar{U}_{0}+\frac{p\left(e^{*}\right)}{p^{\prime}\left(e^{*}\right)}\left[\frac{\partial \bar{U}_{0}}{\partial e}\left(1-p\left(e^{*}\right)\right)+\frac{\partial \bar{U}_{1}}{\partial e} p\left(e^{*}\right)\right] \leq-\bar{U}
$$

where $\bar{U}_{0} \equiv \sum_{i} U_{0}\left(w-\beta_{i}, e^{*}\right) Q_{i}, \frac{\partial \bar{U}_{0}}{\partial e} \equiv \sum_{i} \frac{\partial U_{0}\left(w-\beta_{i}, e^{*}\right)}{\partial e} Q_{i}$, etc. Since (7b) is not necessarily a convex constraint, it appears that randomization may be desirable. Since the analog to (6a) for nonseparable utility is a convex constraint, if constraint (7b) is also convex, then by Lemma 1 randomization is undesirable, i.e.:

Proposition 3. A sufficient condition for ex post randomization to be undesirable is that $\varphi$ be convex in $y_{0}$ and $y_{1}$ jointly.

Proposition 3 has an immediate corollary.

Corollary 1. If $\frac{\partial^{3} U_{0}}{\partial e \partial y_{0}^{2}} \leq 0$ and $\frac{\partial^{3} U_{1}}{\partial e \partial y_{1}^{2}} \leq 0$, then ex post randomization is undesirable.

Note that Proposition 2 is a special case of Corollary $1 .{ }^{9}$

Deriving necessary and sufficient conditions for randomization to be desirable is difficult. A large randomization may be desirable, even when a small one is not, and the desirability of large randomizations depends on global rather than local properties of the functions $U_{0}(\cdot), U_{1}(\cdot)$, and $p(\cdot)$, as well as the parameters $w$ and $d .{ }^{10}$ Determining necessary and sufficient conditions for a local randomization to be desirable is presumably possible, but since efficient randomization may entail three random policies (recall Proposition 1), doing so would be algebraically very tedious. As a result, we shall adopt the more modest goal of deriving a sufficient condition for a local randomization of the payout, while holding the premium fixed, to be desirable. ${ }^{11}$

A necessary condition for a local randomization of the payout with two random policies to be desirable is that a small, mean-preserving spread in $\alpha$, while holding $\beta$ fixed, increase effort. If this condition is not satisfied, then the randomization not only lowers expected utility, by exposing the individual to more risk, but also decreases profits by increasing the probability of accident. From first principles or from Rothschild and Stiglitz (1970) it is straightforward to show that this randomization stimulates effort if and only if

$$
p^{\prime} \frac{\partial^{2} U_{1}}{\partial y_{1}^{2}}+p \frac{\partial^{3} U_{1}}{\partial e \partial y_{1}^{2}}>0 .
$$

A similar necessary condition is that a small, expected-utility-preserving spread in $\alpha$, while holding $\beta$ fixed, increase effort. If this condition is not satisfied, the randomization unambiguously decreases profits; not only does the probability of accident rise, but also compensating the individual for the risk he faces is costly. From first principles or from Diamond and Stiglitz (1974), one obtains that this randomization stimulates effort if and only if

$$
p \frac{\partial^{3} U_{1}}{\partial e \partial y_{1}^{2}}-p \frac{\partial^{2} U_{1}}{\partial e \partial y_{1}}\left[\frac{\partial^{2} U_{1} / \partial y_{1}^{2}}{\partial U_{1} / \partial y_{1}}\right]>0
$$

\footnotetext{
${ }^{9}$ Since they are expressed in terms of the characteristics of the constraint set, Propositions 2 and 3, as well as Corollary 1 , apply to any $(\alpha, \beta)$ (and the associated levels of $\bar{U}$ and $e$ ) and not just to profit-maximizing $\alpha$ and $\beta$ associated with a given level of expected utility. The same remark applies to Proposition 4.

${ }^{10}$ Furthermore, with nonseparable utility, effort need not be a continuous function of the contract parameters. We have chosen not to treat this complication.

${ }^{11}$ We have considered other two-dimensional perturbations: (i) randomizing the premium, while holding the payout fixed; and (ii) randomizing the payout, while varying the premium to maintain profits constant, and investigating the change in expected utility.
} 
A sufficient condition for a local randomization of the payout to be desirable is that at the deterministic optimum (denoted by *) a small, expected-utility-preserving spread in $\alpha$, while holding $\beta$ fixed, increase effort by enough that profits are increased. To calculate this we provide a payout of $\alpha+\Delta$ half the time and $\alpha-\Delta$ half the time and adjust $\alpha$ to keep expected utility constant, and ascertain whether doing so increases profits. Define $\hat{\alpha}\left(\Delta ; \beta^{*}, u^{*}\right)$ to be the value of $\alpha$ generated by this procedure, $\hat{e}\left(\hat{\alpha}, \Delta ; \beta^{*}\right)$ to be the associated level of effort, and $\hat{\Pi}\left(\hat{\alpha}, \Delta ; \beta^{*}\right) \equiv(1-p(\hat{e})) \beta^{*}-p(\hat{e}) \hat{\alpha}$. Furthermore, evaluate all derivatives at the deterministic optimum. Now, $d \hat{\Pi} / d \Delta=(\partial \hat{\Pi} / \partial \Delta)+\partial \hat{\Pi} / \partial \alpha(d \hat{\alpha} / d \Delta)$. Since the randomization is small, $d \hat{\Pi} / d \Delta=d \hat{\alpha} / d \Delta=0$. Hence, it is necessary to examine second derivatives. Specifically, the local randomization is desirable if and only if $d^{2} \hat{\Pi} / d \Delta^{2}>0$. Tedious manipulation gives

$$
\frac{d^{2} \hat{\Pi}}{d \Delta^{2}}=\frac{p^{\prime}(\alpha+\beta)}{E U_{e e}}\left(p \frac{\partial^{3} U_{1}}{\partial e \partial y_{1}^{2}}-p \frac{\partial^{2} U_{1}}{\partial e \partial y_{1}}\left(\frac{\partial^{2} U_{1} / \partial y_{1}^{2}}{\partial U_{1} / \partial y_{1}}\right)\right)+p \frac{\partial^{2} U_{1} / \partial y_{1}^{2}}{\partial U_{1} / \partial y_{1}}
$$

where

$$
E U_{e e}=-p^{\prime \prime}\left(U_{0}-U_{1}\right)-2 p^{\prime}\left(\frac{\partial U_{0}}{\partial e}-\frac{\partial U_{1}}{\partial e}\right)+\frac{\partial^{2} U_{0}}{\partial e^{2}}(1-p)+\frac{\partial^{2} U_{1}}{\partial e^{2}} p<0
$$

from the second-order conditions of the individual's effort choice problem.

Proposition $4 .^{12}$ A sufficient condition for a local randomization of the payout to be desirable, with the premium held fixed, is that $d^{2} \hat{\Pi} / d \Delta^{2}>0$, with the expression for $d^{2} \hat{\Pi} / d \Delta^{2}$ being given in (9).

With separable utility this condition cannot be satisfied, but theory imposes no natural restrictions on the values of $\frac{\partial^{2} U}{\partial e \partial y}$ or $\frac{\partial^{3} U}{\partial e \partial y^{2}}$; hence, it appears that randomization can be desirable. $^{13}$

The result is depicted in Figure 1. In the neighborhood of the deterministic optimum, $\frac{\partial \Pi}{\partial \alpha}<0$ (otherwise, both profits and expected utility could be increased with a rise in $\alpha$ ). Since also both the indifference curve and isoprofit curve through the deterministic optimum have zero slope in $\Delta-\alpha$ space at the deterministic optimum, randomization is desirable if the isoprofit curve has greater positive curvature than the indifference curve.

Ex ante randomization. The desirability of ex ante randomization depends on quite a different set of considerations.

Define $E U(\Pi)$ to be the maximum expected utility as a function of the insurer's (expected ) profit per client, $\Pi$, when only deterministic contracts are admitted. The desirability of ex ante randomization depends on the curvature properties of $E U(\Pi)$. This is shown in Figure 2. Suppose, for the sake of argument, that the solution to the monopoly insurer's problem with deterministic contracts occurs at $C$, with profit per contract of $\Pi_{C}$. Now allow ex ante randomization. Since the realization of the random variable occurs before the individual makes his effort decision, for any level of profit there is a unique expected-utilitymaximizing policy. This implies that if the profit-maximizing contract contains more than one policy, each must have a different level of profits associated with it. Suppose that the insurer offers two ex ante random policies in his contract, policy $A$ with profits $\Pi_{A}<\Pi_{C}$ and policy $B$ with profits $\Pi_{B}>\Pi_{C}$, with the probability weights chosen so that the expected

${ }^{12}$ This proposition has been proved previously by Gjesdal (1982, Proposition 3) for the continuum of outcomes case. Our proof, however, is new.

${ }^{13}$ Gjesdal (1982, pp. 382-383) provides an example in which ex post randomization is desirable. 


\section{FIGURE 1}

EX POST RANDOMIZATION WITH MORAL HAZARD (A PREFERRED TO B)

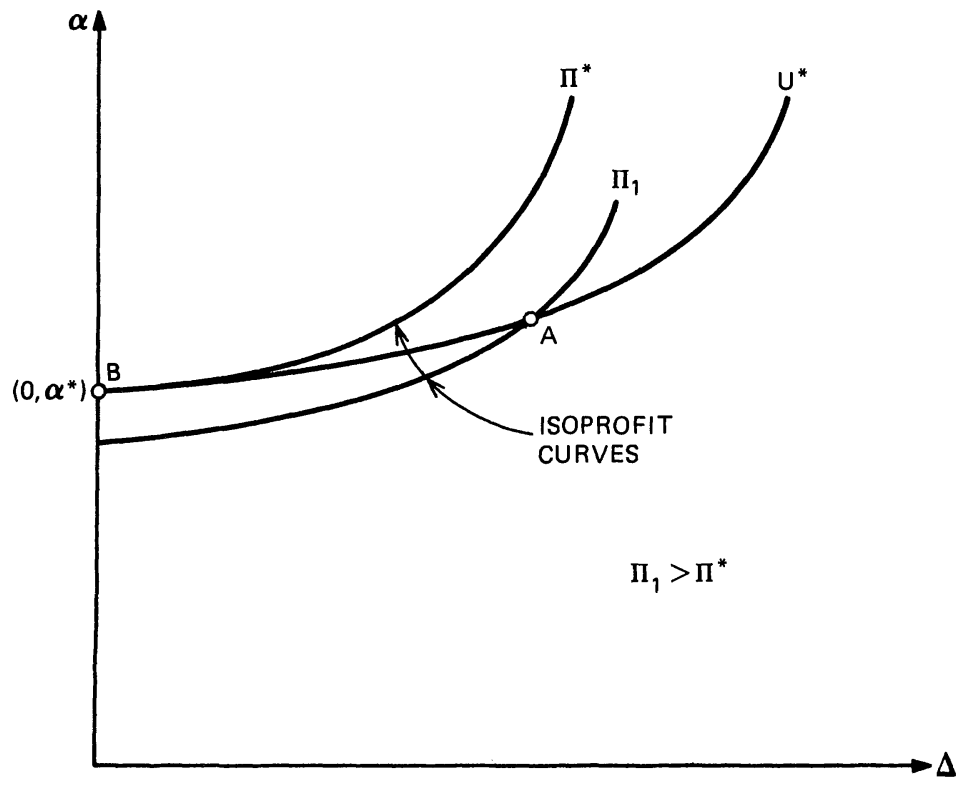

profit from the pair of contracts is $\Pi_{C}$. It is evident from the diagram that, as drawn, this ex ante randomization increases expected utility and is therefore desirable. It is also evident that the desirability of ex ante randomization stems from the convexity of $E U(\Pi)$ near $C$, and that no more than two random policies are needed in a profit-maximizing contract with ex ante randomization.

We formalize this intuition in a series of propositions.

\section{FIGURE 2}

EX ANTE RANDOMIZATION WITH MORAL HAZARD (WEIGHTED AVERAGE OF A AND B

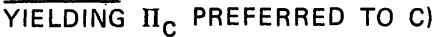

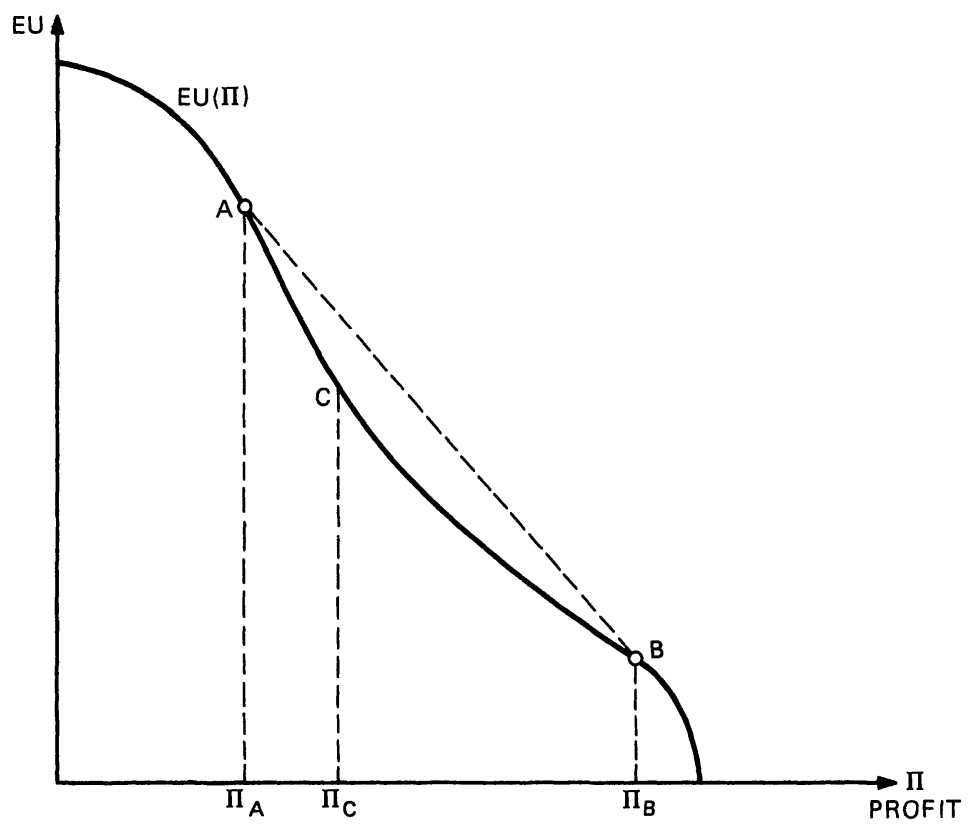


Proposition 5. If $E U(\Pi)$ is concave, ex ante randomization is never desirable.

Proposition 6. If $E U(\Pi)$ is convex at the level of profits at the deterministic monopoly insurer's profit maximum, then ex ante randomization is desirable. ${ }^{14}$

Proposition 7. With ex ante randomization no more than two random policies are required in the profit-maximizing insurance contract.

In the absence of any moral-hazard problem, expected utility is a concave function of $\Pi$ because of diminishing marginal utility. But with moral hazard, as $\Pi$ changes, effort changes. In the normal case, as profit increases (decreases), the individual will expend more (less) effort at accident avoidance. If the absolute value of the individual's effort response is greater for increases in profits than for decreases (in a sense to be made more precise below), expected utility may be a convex function of $\Pi$ and ex ante randomization may be desirable.

It should be clear from Figure 2 that convexity of $E U(\Pi)$ at $C$ is not a necessary condition for ex ante randomization to be desirable; in particular, nonlocal randomization may be desirable even when $E U(\Pi)$ is concave at $C$. The desirability of nonlocal randomization is difficult to characterize because it depends on global rather than local properties of the event-contingent utility functions and the probability of accident function. In what follows we shall therefore consider only local randomizations.

To simplify we hold $\beta$ fixed at its level in the absence of randomization ( since if expected utility can be increased via ex ante randomization with $\beta$ fixed, it can $a$ fortiori be increased with $\beta$ variable) and treat the special case of a separable, event-independent utility function (1b). Recall that from the individual's maximization problem without randomization, we can express effort as a function of the contract parameters; i.e., $e=e(\alpha, \beta)$. Now $\Pi=\beta(1-p)-\alpha p$. Thus, we may write

$$
e(\alpha, \beta)=e\left(\frac{\beta(1-p)-\Pi}{p}, \beta\right)=\hat{e}(\Pi, \beta) .
$$

Substitute this into the expression for expected utility,

$$
\begin{aligned}
V(\Pi, \beta, \hat{e}(\Pi, \beta)) & \equiv \mathcal{V}(\Pi, \beta) \\
& \equiv(1-p(\hat{e})) u(w-\beta)+p(\hat{e}) u\left(w-d+\frac{\beta(1-p(\hat{e}))-\Pi}{p(\hat{e})}\right)-\hat{e} .
\end{aligned}
$$

Ex ante randomization is desirable if $\partial^{2} \mathcal{V} / \partial \Pi^{2}>0$, evaluated at the deterministic optimum. The following expression for $\partial^{2} \mathcal{V} / \partial \Pi^{2}$ is derived in Appendix A:

$$
\begin{aligned}
\frac{\partial^{2} \mathcal{V}}{\partial \Pi^{2}}=\frac{1}{Z_{e}^{3}}\left\{Z _ { e } \left(V_{\Pi \Pi} Z_{e}^{2}-2 V_{e \Pi} Z_{e} Z_{\Pi}+\right.\right. & \left.V_{e e} Z_{\Pi}^{2}\right) \\
& \left.-V_{e}\left(Z_{\Pi \Pi} Z_{e}^{2}-2 Z_{e \Pi} Z_{e} Z_{\Pi}+Z_{e e} Z_{\Pi}^{2}\right)\right\},
\end{aligned}
$$

where subscripts denote partial derivatives and

$$
Z(\Pi, \beta, \hat{e}) \equiv-p^{\prime}(\hat{e})\left[u(w-\beta)-u\left(w-d+\frac{\beta(1-p(\hat{e}))-\Pi}{p(\hat{e})}\right)\right]-1 .
$$

We have thus established the following.

Proposition 8. A sufficient condition for ex ante randomization to be desirable is that the expression on the right-hand side of (12), evaluated at the deterministic optimum, be positive.

${ }^{14}$ This proposition is similar to Theorem 3 of Fellingham, Kwon, and Newman (1984). 
After substituting the expressions for the various partial derivatives in (12), the resulting expression is extremely messy. Nevertheless, the following argument establishes that $\frac{\partial^{2} \mathcal{V}}{\partial \Pi^{2}}$ can be positive: The only place where $p^{\prime \prime \prime}$ enters the expression is through $Z_{e e}$. The full term containing $p^{\prime \prime \prime}$ is $-V_{e} Z_{I I}^{2}\left(-p^{\prime \prime \prime}\left(u_{0}-u_{1}\right)\right) / Z_{e}^{3}$. Since $V_{e}>0, Z_{e}<0$, and $u_{0}-u_{1}>0$, then if $p^{\prime \prime \prime}$ is negative and sufficiently large in absolute value, $\partial^{2} V / \partial \Pi^{2}$ will be positive. ${ }^{15}$ The reason for the complexity of the result is that what is relevant is the extent to which successive increases in $\Pi$ lead to increases in effort (and how these increases in effort lead to increases in the likelihood of a loss). Since the level of effort depends on first derivatives ( say of $p(e)$ ), changes in levels depend on second derivatives, and differences in changes in levels (which are critical for ascertaining the convexity or concavity of profit as a function of $\Pi$ ) depend on third derivatives.

To simplify we have considered ex post and ex ante randomization in isolation. It should be evident that there are circumstances in which it is desirable to use both forms of randomization simultaneously.

\section{Adverse selection}

- It seems plausible that randomization can be desirable when adverse selection is present, by altering the position of the self-selection locus. To investigate this we treat the case where there are two groups in the population, one high-risk $(H)$ and one low-risk $(L)$.

The exposition of the adverse-selection case is simplified if we analyze the desirability of randomization in the context of a competitive insurance market, though as in the moralhazard section, the results carry over to other market forms. In particular, we start with the competitive equilibrium with deterministic contracts and investigate under what circumstances a single insurer can increase his profits by randomizing his insurance contract. Suppose that the competitive equilibrium with deterministic contracts entails group $H$ 's receiving contract $\left(\hat{\alpha}^{H}, \hat{\beta}^{H}\right)$ and group $L$ 's receiving contract $\left(\hat{\alpha}^{H}, \hat{\beta}^{H}\right)$, as depicted in Figure 3. Group $H$ 's utility is $\hat{U}^{H}$, and group $L$ 's utility is $\hat{U}^{L}$. The basic adverse-selection mechanism in this context should be familiar. If insurers were able to distinguish between individuals in the two groups, the equilibrium contracts would be $\left(\hat{\alpha}^{H}, \hat{\beta}^{H}\right)$ and $\left(\tilde{\alpha}^{L}, \tilde{\beta}^{L}\right)$, providing each group with full and actuarially fair insurance. But with adverse selection, insurers are unable to distinguish between high- and low-risk individuals. As a consequence, if the contracts $\left(\hat{\alpha}^{H}, \hat{\beta}^{H}\right)$ and $\left(\tilde{\alpha}^{L}, \tilde{\beta}^{L}\right)$, were offered, the high-risk group would purchase $\left(\tilde{\alpha}^{L}, \tilde{\beta}^{L}\right)$, which would cause insurers in the aggregate to operate at a loss. If the proportion of the population in the high-risk group is above a certain critical level, which we assume is the case, competitive equilibrium entails $\left(\hat{\alpha}^{H}, \hat{\beta}^{H}\right)$ and $\left(\hat{\alpha}^{L}, \hat{\beta}^{L}\right)$, where $\left(\hat{\alpha}^{L}, \hat{\beta}^{L}\right)$ provides the maximum actuarially fair insurance to the low-risk group that is consistent with the highrisk group's preferring to self-select, i.e., to choose $\left(\hat{\alpha}^{H}, \hat{\beta}^{H}\right)$, which is more expensive but provides more insurance, rather than the contract designed for low-risk individuals. Thus, $\left(\hat{\alpha}^{L}, \hat{\beta}^{L}\right)$, lies on $\beta\left(1-p^{L}\right)-\alpha^{L} p^{L}=0$, "just below" the point where $\hat{U}^{H}$ intersects this zero-profit line. Since the high-risk group prefers $\left(\hat{\alpha}^{H}, \hat{\beta}^{H}\right)$ to those contracts above $\hat{U}^{H}$ in the figure, but not those below, $\hat{U}^{H}$ is called the self-selection locus. We focus on a single insurer in this market, and examine conditions under which this firm can increase its profits by randomizing its contracts, with all other firms' continuing to offer the equilibrium deterministic contracts.

We now consider the use of randomization as a self-selection device. Again, randomization can take on two forms. (Both are set out in Table 1.) In the first the insurer announces two contracts, denoted by $A$ and $B$. If the individual chooses contract $A$, he will be assigned

${ }^{15}$ Fellingham, Kwon, and Newman (1984, pp. 296-297) provide an example in which ex ante randomization is desirable. 
FIGURE 3

ADVERSE SELECTION

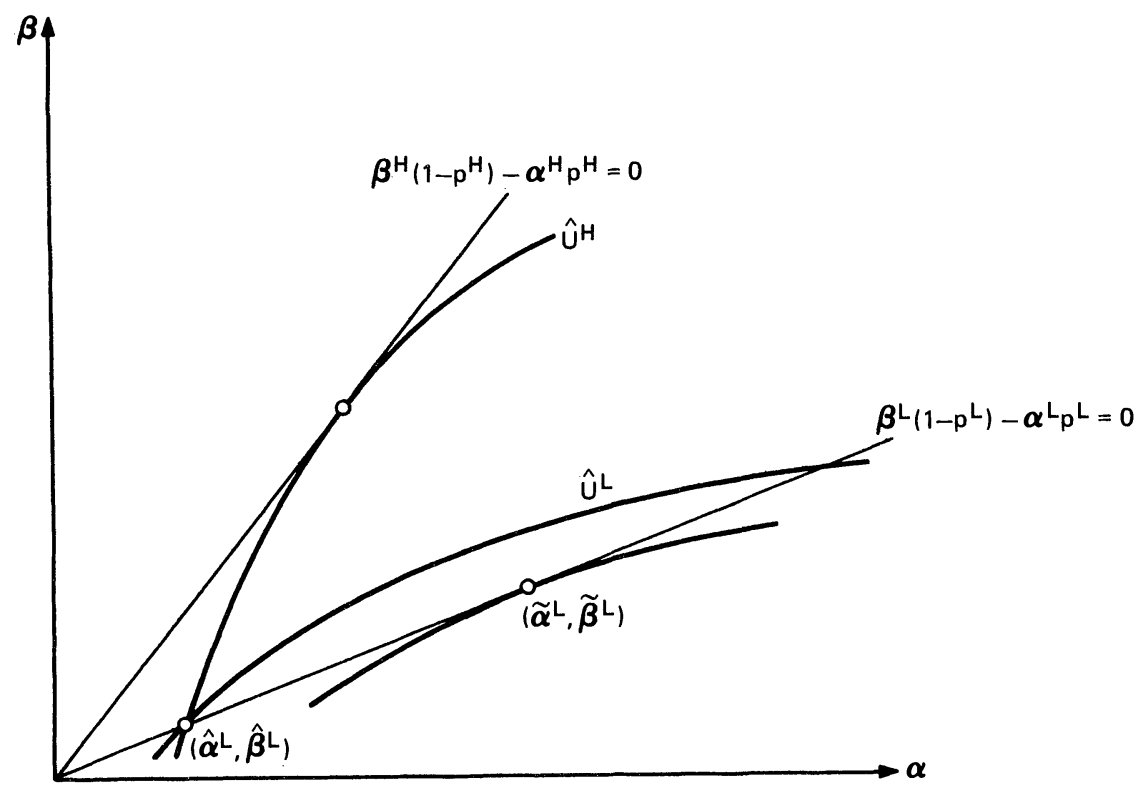

policy ( $\alpha_{i}^{A}, \beta_{i}^{A}$ ) with probability $Q_{i}^{A}$; similarly, if he chooses contract $B$, he will be assigned $\left(\alpha_{i}^{B}, \beta_{i}^{B}\right)$ with probability $Q_{i}^{B}$. The individual reveals his type by his choice of contract. We say that offering such a pair of contracts entails ex post randomization, since the uncertainty is resolved after the individual chooses between the two contracts, i.e., after he has revealed his type. Traditional adverse-selection models can be viewed as treating the case where $A$ and $B$ both consist of a single policy; we ask here, when is this desirable?

In the second form that randomization may take the insurer gives the individual a lottery of policy pairs, $L_{i}=\left[\left(\alpha_{i}^{A}, \beta_{i}^{A}\right),\left(\alpha_{i}^{B}, \beta_{i}^{B}\right), Q_{i}\right], \sum_{i=1}^{I} Q_{i}=1$. The firm then randomly assigns him a policy pair, e.g., $L_{i^{\prime}}$ with probability $Q_{i^{\prime}}$. Having been assigned a policy pair, say $L_{i^{\prime}}$, the individual can then choose between $A_{i^{\prime}}=\left(\alpha_{i^{\prime}}^{A}, \beta_{i^{\prime}}^{A}\right)$ and $B_{i^{\prime}}=\left(\alpha_{i^{\prime}}^{B}, \beta_{i^{\prime}}^{B}\right)$. The

TABLE 1

The Timing of ex ante and ex post Randomization with Adverse Selection

Ex post Randomization

Insurance firm offers two contracts:

$$
\begin{array}{cc}
A & B \\
\left(\alpha_{1}^{A}, \beta_{1}^{A}\right), Q_{1}^{A} & \left(\alpha_{1}^{B}, \beta_{1}^{B}\right), Q_{1}^{B} \\
\left(\alpha_{2}^{A}, \beta_{2}^{A}\right), Q_{2}^{A} & \left(\alpha_{2}^{B}, \beta_{2}^{B}\right), Q_{2}^{B} \\
\vdots & \vdots \\
\left(\alpha_{N}^{A}, \beta_{N}^{A}\right), Q_{N}^{A} & \left(\alpha_{M}^{B}, \beta_{M}^{B}\right), Q_{M}^{B} \\
\sum_{1}^{N} Q_{i}^{A}=1 & \sum_{1}^{M} Q_{i}^{B}=1 .
\end{array}
$$

Individual chooses $A$ or $B$.

Individual randomly assigned insurance policy $\left(\alpha_{i}^{A}, \beta_{i}^{A}\right)$ with probability $Q_{i}^{A}$ if chose $A$, etc.

Uncertainty resolved after individual's choice (of lotteries).
Ex ante Randomization

Insurance firm offers a lottery of policy pairs:

$$
\begin{gathered}
L_{i}=\left[\left(\alpha_{i}^{A}, \beta_{i}^{A}\right),\left(\alpha_{i}^{B}, \beta_{i}^{B}\right), Q_{i}\right] \\
\sum_{1}^{l} Q_{i}=1 .
\end{gathered}
$$

Individual randomly assigned $L_{i}$ with probability $Q_{i}$.

Having been assigned $L_{i}$, individual chooses between

$$
\begin{aligned}
A_{i} & =\left(\alpha_{i}^{A}, \beta_{i}^{A}\right) \text { and } \\
B_{i} & =\left(\alpha_{i}^{B}, \beta_{i}^{B}\right) .
\end{aligned}
$$

Uncertainty resolved before individual's choice of policies. 
individual reveals his type through his choice of $A_{i^{\prime}}$ or $B_{i^{\prime}}$. We say that such a contract entails ex ante randomization, since the uncertainty is resolved before the individual makes his choice, and therefore before he reveals his type. In the standard adverse-selection model the individual is offered a single pair of policies, rather than a lottery of pairs of policies.

We shall use the following additional notation: $n^{k}$, the population of group $k$, $k=H, L ; p^{k}$, the accident probability of group $k ; u_{0}^{k}$, the utility function for group $k$ in the event of no accident; and $u_{1}^{k}$, the corresponding utility function when an accident occurs. Superscript $k(=H, L)$ on a contract parameter indicates that the corresponding contract is designed for group $k$.

We first prove the following.

Proposition 9. The insurer can always do at least as well with ex post randomization, as with ex ante randomization.

Proof. The profit-maximizing ex ante random contract solves

$$
\begin{aligned}
& \max _{\left\{Q_{\|,}\left(\alpha_{t}^{H}, \beta_{i}^{H}\right),\left(c^{i}, \beta_{i}^{L}\right)\right\}} n^{H}\left(\left(1-p^{H}\right) \sum_{i} Q_{i} \beta_{i}^{H}-p^{H} \sum_{i} Q_{i} \alpha_{i}^{H}\right) \\
& +n^{L}\left(\left(1-p^{L}\right) \sum_{i} Q_{i} \beta_{i}^{L}-p^{L} \sum_{i} Q_{i} \alpha_{i}^{L}\right)
\end{aligned}
$$

subject to

$$
\begin{array}{r}
\left(1-p^{H}\right) \sum_{i} u_{0}^{H}\left(w-\beta_{i}^{H}\right) Q_{i}+p^{H} \sum_{i} u_{1}^{H}\left(w-d+\alpha_{i}^{H}\right) Q_{i} \geq \hat{U}^{H} \\
\text { (expected utility constraints) } \\
\left(1-p^{L}\right) \sum_{i} u_{0}^{L}\left(w-\beta_{i}^{L}\right) Q_{i}+p^{L} \sum_{i} u_{1}^{L}\left(w-d+\alpha_{i}^{L}\right) Q_{i} \geq \hat{U}^{L} \\
\left(1-p^{H}\right)\left(u_{0}^{H}\left(w-\beta_{i}^{H}\right)-u_{0}^{H}\left(w-\beta_{i}^{L}\right)\right)+p^{H}\left(u_{1}^{H}\left(w-d+\alpha_{i}^{H}\right)\right. \\
\left.-u_{1}^{H}\left(w-d+\alpha_{i}^{L}\right)\right) \geq 0 \quad \forall i \quad \text { (self-selection constraints) } \\
\sum_{i} Q_{i}=1, \quad Q_{i} \geq 0 \quad \forall i
\end{array}
$$

Consider the profit-maximization problem identical to (13) in all respects except that the set of constraints (13c) is replaced by

$$
\begin{aligned}
\left(1-p^{H}\right) \sum_{i}\left(u_{0}^{H}\left(w-\beta_{i}^{H}\right)-u_{0}^{H}\left(w-\beta_{i}^{L}\right)\right) Q_{i}+p^{H} \sum_{i}\left(u_{1}^{H}\left(w-d+\alpha_{i}^{H}\right)\right. & \\
& \left.-u_{1}^{H}\left(w-d+\alpha_{i}^{L}\right)\right) Q_{i} \geq 0 .
\end{aligned}
$$

We term this the modified-maximization problem. Since this constraint is weaker than the set of constraints (13c), profits are at least as high for the modified-maximization problem as for (13). But the modified maximization problem is that of an insurer who undertakes ex post randomization. Q.E.D.

We have established that ex ante randomization is unnecessary by showing that ex post randomization is always at least as profitable. If there are restrictions on ex post randomization, ex ante randomization may still be desirable. We shall not, however, attempt to determine conditions under which ex ante randomization is, in fact, desirable.

Proposition 9 is useful because it enables us to focus on ex post randomization. Ex post randomization of the high-risk group's contract is unambiguously harmful since it reduces the profitability of the high-risk group's contract, while leaving the low-risk group's contract unchanged. Ex post randomization of the low-risk group's contract, meanwhile, 
has two possibly off-setting effects. On the one hand, the low-risk individuals must be compensated for the increased risk they then face. On the other hand, such randomization may weaken the self-selection constraint. If high-risk individuals are very risk averse, they will shy away from the randomized contract and allow the low-risk individuals to obtain more insurance on average than they otherwise would. The propositions that follow capture this intuition.

We start by considering the case where high- and low-risk individuals have the same tastes (event-contingent utility functions). Let $b$ be the certainty-equivalent insurance premium corresponding to the ex post random contract $\left\{\alpha_{i}, \beta_{i}, Q_{i}\right\}$, defined implicitly by

$$
u_{0}(w-b) \equiv \sum_{i} u_{0}\left(w-\beta_{i}\right) Q_{i}
$$

and let $a$ be the certainty-equivalent insurance payout, defined implicitly by

$$
u_{1}(w-d+a) \equiv \sum_{i} u_{1}\left(w-d+\alpha_{i}\right) Q_{i}
$$

The expected utility for an individual in group $k$ is, using (14),

$$
\begin{aligned}
E U^{k} & =\left(1-p^{k}\right)\left(\sum_{i} u_{0}\left(w-\beta_{i}\right) Q_{i}\right)+p^{k}\left(\sum_{i} u_{1}\left(w-d+\alpha_{i}\right) Q_{i}\right) \\
& =\left(1-p^{k}\right) u_{0}(w-b)+p^{k} u_{1}(w-d+a) .
\end{aligned}
$$

Thus, expected utility can be expressed in terms of the certainty-equivalent premium and payout corresponding to any ex post random contract. Meanwhile, we can write the selfselection constraint as

$$
\begin{aligned}
& \left(1-p^{H}\right)\left(u_{0}\left(w-b^{H}\right)-u_{0}\left(w-b^{L}\right)\right) \\
& +p^{H}\left(u_{1}\left(w-d+a^{H}\right)-u_{1}\left(w-d+a^{L}\right)\right) \geq 0,
\end{aligned}
$$

where $\left(a^{k}, b^{k}\right)$ is the certainty-equivalent of the random contract $\left\{\alpha_{i}^{k}, \beta_{i}^{k}, Q_{i}^{k}\right\}$ designed for group $k$. Next, define $\bar{\beta}^{k} \equiv \sum_{i} Q_{i}^{k} \beta_{i}^{k}$ to be the average premium in the random contract designed for group $k$, and $\bar{\alpha}^{k} \equiv \sum_{i} Q_{i}^{k} \alpha_{i}^{k}$ to be the corresponding average payout. Risk aversion implies that $a^{k} \leq \bar{\alpha}^{k}$ and $b^{k} \geq \bar{\beta}^{k}$, with at least one of the inequalities being strict if the corresponding contract is randomized.

The group $k$ expected utility constraint, using (15), may be written as

$$
\left(1-p^{k}\right) u_{0}\left(w-b^{k}\right)+p^{k} u_{1}\left(w-d+a^{k}\right) \geq \hat{U}^{k}, \quad k=H, L .
$$

We have shown that with a random contract the self-selection constraint and the expected utility constraints can be expressed in terms of the certainty-equivalent premium and payout of that contract. The insurer's profit-maximization problem, with ex post randomization, may be written as

$$
\max _{\substack{\left\{a^{k}, \bar{\alpha}^{k}, b^{k}, \bar{\beta}^{k}\right\} \\ k=H, L}} \sum_{\substack{k=H, L\\}} n^{k}\left(\left(1-p^{k}\right) \bar{\beta}^{k}-p^{k} \bar{\alpha}^{k}\right)
$$

subject to (a) (16) (the self-selection constraint), (b) (17) (the expected utility constraints), and (c) $a^{k} \leq \bar{\alpha}^{k}, b^{k} \geq \bar{\beta}^{k}$. From this formulation, it is evident that the profit-maximizing contract pair has the properties $\bar{\alpha}^{k}=a^{k}$ and $\bar{\beta}^{k}=b^{k}$ for $k=H, L$, and therefore that $e x$ post randomization is undesirable.

This result is stated in the following. ${ }^{16}$

${ }^{16}$ It can also be proved, rather obviously, that with adverse selection and two groups, randomization is never desirable if the high-risk group is less risk averse than the low-risk group. 
Proposition 10. With adverse selection and two groups that differ in risk but not in tastes, ex post randomization is never desirable.

The intuition underlying this result can be seen from Figure 4 . The premiums and payouts of the deterministic equilibrium contracts equal their corresponding certainty equivalents, i.e., $\left(\hat{\alpha}^{H}, \hat{\beta}^{H}\right)=\left(a^{H}, b^{H}\right)$ and $\left(\hat{\alpha}^{L}, \hat{\beta}^{L}\right)=\left(a^{L}, b^{L}\right)$. Thus, the equilibrium with deterministic contracts can be represented in $a-b$ space; see Figure 4.

Now consider randomizing the contract for the low-risk group while holding $\hat{U}^{H}$ and $\hat{U}^{L}$ fixed. Since the randomization does not alter the position of the self-selection constraint in $a-b$ space, the low-risk group's certainty-equivalent policy remains $\left(\hat{a}^{L}, \hat{b}^{L}\right)$. Since with randomization $\bar{\alpha}^{L} \geq a^{L}$ and $\bar{\beta}^{L} \leq b^{L}$, with at least one of the inequalities holding strictly, then $\bar{\beta}^{L}\left(1-p^{L}\right)-\bar{\alpha}^{L} p^{L}<b^{L}\left(1-p^{L}\right)-a^{L} p^{L}=0$. Thus, the randomization reduces profits on the low-risk group's policy, while leaving profits on the high-risk group's policy unaffected.

We now examine the situation where individuals differ in terms of both risk class and tastes for risk. We derive a condition under which a small amount of randomization is desirable.

We randomize the contract for low-risk individuals in such a way that: ${ }^{17}$ (i) there are two policies, both with probability .5; (ii) low-risk individuals' utility is the same with either policy and equals their certainty utility; and (iii) the profit level on the low-risk contract is maintained. Randomization will then be desirable if (and only if) it lowers the utility of the high-risk individuals when they hold the low-risk contract, since if this happens, the self-selection constraint is relaxed, and the low-risk contract can then be adjusted to increase its profitability. To simplify the algebra, we assume that both groups' utility functions are event-independent.

This randomization satisfies the conditions:

$$
\left(1-p^{L}\right) u^{L}\left(w-\beta_{1}^{L}\right)+p^{L} u^{L}\left(w-d+\alpha_{1}^{L}\right)=\hat{U}^{L}
$$

FIGURE 4

RANDOMIZATION UNDESIRABLE WHEN BOTH GROUPS HAVE SAME TASTES

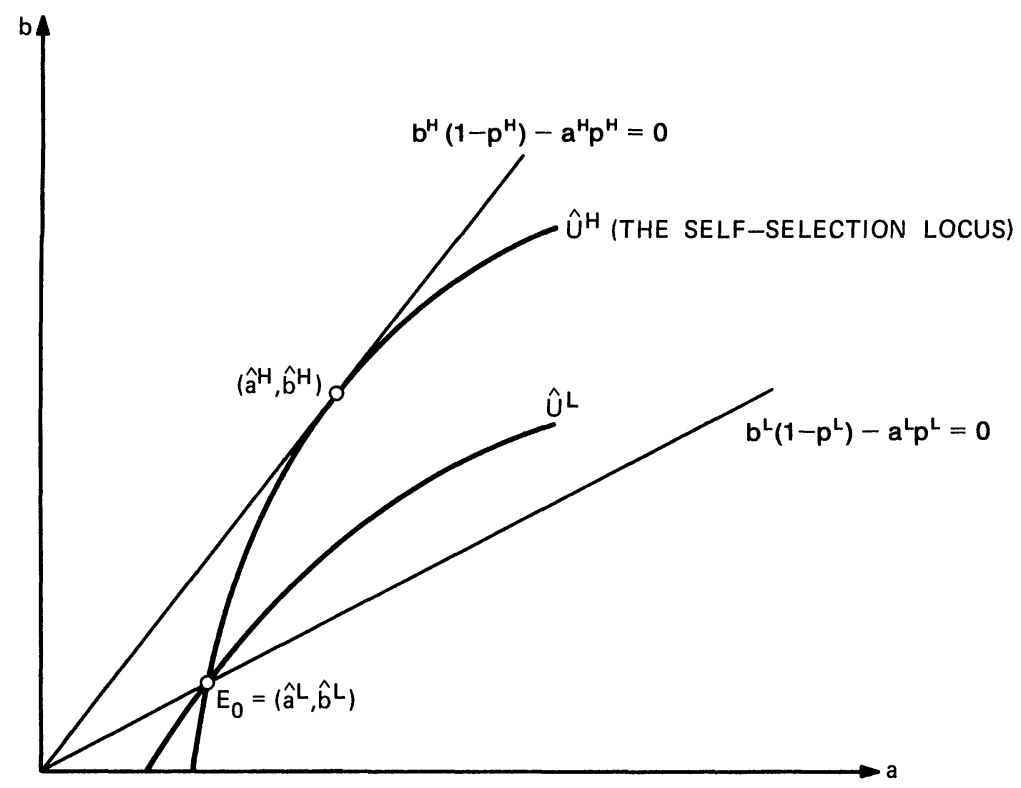

${ }^{17}$ We would like to thank Stephen Slutsky and John Hamilton for suggesting this randomization procedure to us in another context. 


$$
\begin{aligned}
\left(1-p^{L}\right) u^{L}\left(w-\beta_{2}^{L}\right)+p^{L} u^{L}\left(w-d+\alpha_{2}^{L}\right) & =\hat{U}^{L} \\
\left(1-p^{L}\right)\left(\beta_{1}^{L}+\beta_{2}^{L}\right)-p^{L}\left(\alpha_{1}^{L}+\alpha_{2}^{L}\right) & =0 .
\end{aligned}
$$

The expected utility of a high-risk individual when he purchases a low-risk contract is

$$
\begin{aligned}
E U^{H}=.5\left\{( 1 - p ^ { H } ) \left(u^{H}\left(w-\beta_{1}^{L}\right)+\right.\right. & \left.u^{H}\left(w-\beta_{2}^{L}\right)\right) \\
& \left.+p^{H}\left(u^{H}\left(w-d+\alpha_{1}^{L}\right)+u^{H}\left(w-d+\alpha_{2}^{L}\right)\right)\right\} .
\end{aligned}
$$

We show in Appendix B that the first-order effect of an infinitesimal randomization of this nature is zero; i.e., $d E U^{H} / d \alpha_{1}^{L}=0$, evaluated at the deterministic equilibrium. To ascertain the desirability of a small randomization, we investigate the sign of $d^{2} E U^{H} / d\left(\alpha_{1}^{L}\right)^{2}$ at the deterministic equilibrium. Define:

$$
\begin{aligned}
& \theta=\left(\frac{p^{L}}{1-p^{L}}\right) \frac{\left(u_{1}^{L}\right)^{\prime}}{\left(u_{0}^{L}\right)^{\prime}}, \quad \begin{array}{l}
\text { the slope of the low-risk indifference curve } \\
\text { with the contract }\left(\alpha_{1}^{L}, \beta_{1}^{L}\right) ;
\end{array} \\
& r^{k}=\frac{\left(u_{0}^{k}\right)^{\prime}}{\left(u_{1}^{k}\right)^{\prime}}, \quad \begin{array}{l}
\text { the ratio of the marginal utility of consumption } \\
k \text { with the contract }\left(\alpha_{1}^{L}, \beta_{1}^{L}\right) ;
\end{array} \\
& A_{j}^{k} \equiv-\frac{\left(u_{j}^{k}\right)^{\prime \prime}}{\left(u_{j}^{k}\right)^{\prime}}, \quad \begin{array}{l}
\text { the (local) coefficient of absolute risk aversion for group } k \\
\text { and event } j \text { with the contract }\left(\alpha_{1}^{L}, \beta_{1}^{L}\right) ; \text { and }
\end{array} \\
& \eta \equiv \frac{\left(1-p^{H}\right) p^{L}}{\left(1-p^{L}\right) p^{H}}
\end{aligned}
$$

Note that: (i) $\eta<1$; (ii) with risk aversion and incomplete insurance, $r^{k}<1$ for $k=H, L$; and (iii) the greater the degree of risk aversion, the smaller is $r^{k}$ (when holding $\left(\alpha_{1}^{L}, \beta_{1}^{L}\right)$ constant). Then we can show (we explain the procedure of the derivation in Appendix B) that for $A_{j}^{k} \gg 0$ (finitely greater than zero) for $j=0,1 ; k=H, L$ :

$$
\begin{aligned}
\operatorname{sgn}\left[\frac{d^{2} E U^{H}}{d\left(\alpha_{1}^{L}\right)^{2}}\right]=\operatorname{sgn}\left[-A_{0}^{H}\left(\eta r^{H} \theta\left(1-r^{L}\right)\right)-\right. & A_{1}^{H} r^{L}\left(1-r^{L}\right) \\
& \left.+A_{0}^{L} r^{L} \theta\left(1-\eta r^{H}\right)+A_{1}^{L} r^{L}\left(1-\eta r^{H}\right)\right] .
\end{aligned}
$$

Let $\underline{A}^{k}$ denote the minimum of the coefficient of absolute risk aversion for $u^{k}(y)$ with $y \in[w-d, w]$, and $\bar{A}^{k}$ the corresponding maximum, $k=H, L$. Propositions 11 and 12 then follow from the derivation of (23).

Proposition 11. For any $\left(w, d, u^{L}(\cdot), p^{H}, p^{L}\right)$ such that $d \gg 0, p^{H} \gg p^{L}$, and $\bar{A}^{L}$ finite, there is a finite number $\hat{A}^{H}$ such that with $\underline{A}^{H}>\hat{A}^{H}$, ex post randomization is desirable.

The intuition for this result is straightforward. When a high-risk individual is infinitely risk-averse, he will care only about the worst possible outcome. Since the randomization worsens the worst possible outcome when the low-risk contract is bought, it reduces the utility of the high-risk individual when he purchases the low-risk contract, which relaxes the self-selection constraint.

Proposition 12. For any $\left(w, d, u^{H}(\cdot), p^{H}, p^{L}\right)$ such that $d \gg 0$, and $p^{H} \gg p^{L}$, there is a finite number $\hat{A}^{L}$ such that with $\bar{A}^{L}<\hat{A}^{L}$, ex post randomization is desirable.

Consider the extreme case where $\hat{A}^{L}$ is close to zero. Then the randomization undertaken is almost a randomization along the low-risk zero-profit line. Because the high-risk group is risk-averse, its expected utility increases less from the increase in insurance in one policy than its expected utility decreases from the almost same-sized decrease in insurance in the other policy. Hence, the randomization loosens the self-selection constraint. 
Note that none of the above results depends on constancy of absolute risk aversion and that finite randomizations may be Pareto-improving even when infinitesimal randomizations are not.

We have examined the circumstances under which randomization is desirable with either moral hazard or adverse selection, and have explained the results. Future research should extend these results to the general situation where both moral hazard and adverse selection are present.

\section{Discussion}

In the previous two sections we used standard assumptions to derive necessary or sufficient conditions for the randomization of insurance contracts to be desirable. We investigated the desirability of randomization by analyzing whether randomization could increase the profits of an insurer faced with expected utility constraints. It bears repeating that if each insurer can observe his clients' total purchases of insurance, competitive equilibrium ${ }^{18}$ coincides with the solution to the monopoly insurer's problem when the expected utility levels are those that would obtain in competitive equilibrium. Hence, all our results apply to both the competitive-equilibrium and monopoly-insurer cases.

In the absence of relevant empirical work, it is not possible to say how stringent the conditions we derived for randomization to be desirable in fact are. None of our arguments implies that randomization is "normally" undesirable, however. Thus, it is remarkable that randomization of insurance contracts is not in fact observed. This statement needs to be qualified somewhat since the seeming capriciousness of the tax collector and insurance adjustor could be veiled forms of randomization. ${ }^{19}$ Nevertheless, we know of no explicit random contracts in competitive insurance markets. How can we explain this apparent discrepancy between theory and fact?

Along with the possibility that the conditions for randomization are never met, we have come up with six reasons why randomization might not occur so frequently as the theory suggests. Each of these reasons leads one to question the appropriateness of some of the assumptions underlying our analysis and more generally underlying much of the literature on incentives and contracts, particularly that focusing on nonlinear contracts.

(1) Firms may not have discovered the advantages of randomization or customers may be reluctant to purchase random insurance policies since they do not understand them. To the extent that this explanation is valid, one must question the appropriateness of assuming that the contracting parties exhibit unbounded rationality.

(2) A related argument is that it is costly to write down and enforce complex contracts. This argument is consistent with the observation that nonstochastic contracts are almost invariably considerably less complex than contract theory predicts. If this argument carries force, then contract theory should treat the costs of complexity.

Simple contracts may be observed not only because the costs of complexity are high, but also because the benefits are low. ${ }^{20}$

${ }^{18}$ By competitive equilibrium, we mean a Nash equilibrium in contracts with free entry and exit. In the case of adverse selection this statement applies only when competitive equilibrium exists. See Rothschild and Stiglitz (1976) for a discussion of nonexistence of competitive equilibrium in this context.

${ }^{19}$ We consider this unlikely, however. A more plausible explanation for the randomness of tax audits is that decreasing the probability of audit, while holding the expected fine constant, reduces administrative costs.

${ }^{20}$ In moral-hazard theory it is assumed that insurance companies know the accident technology and consumers' tastes perfectly, and in adverse-selection theory that they know consumers' tastes and the composition of the population perfectly. Intuition suggests that the more imperfect is insurers' knowledge, the smoother the optimal contract and the smaller the gains from complexity. 
(3) Even in those circumstances where randomization of insurance contracts is desirable, insured individuals would obtain insurance against the randomization if they could. They would perceive the benefits of randomization insurance, but because they act noncooperatively would neglect that, in response to everyone's purchasing the randomization insurance, accident insurance companies would be forced to offer less attractive contracts to break even. Indeed, full randomization insurance would completely neutralize the effects of randomization of the accident insurance contract, and so accident insurance firms would have no incentive to randomize. The analysis assumed away such randomization insurance. ${ }^{21}$ But with limited observability, which underlies both moral hazard and adverse selection, the assumption may be unwarranted. ${ }^{22} \mathrm{~A}$ more satisfactory analysis would describe the economy in such a way that the markets that are present and the contracts that are enforceable are derived rather than assumed.

(4) In some contexts (e.g., the draft in the United States) lotteries are commonly regarded as fair, in other contexts as unfair (horizontal equity). While the persuasive modelling of fairness and horizontal equity has proved elusive, it may be that consumers would find random insurance policies unattractive because they view them as unfair.

(5) For other familiar reasons one may question the appropriateness of using von NeumannMorgenstern expected utility theory (Machina, 1987).

(6) Such contracts may not be employed because individuals may not trust their insurers to randomize in the manner specified in their contracts. To know whether a firm is complying with the contract, an individual must know not only which policy he has been assigned, but also which policies have been assigned to all the other clients. Even if he were able to verify that the firm assigned the policies with the contractually specified probabilities, the firm could still cheat by assigning the policies in a systematic way, e.g., to friends or to those offering kickbacks. Whether reputation would be an effective enforcement mechanism in this context is moot.

A final point is that precluding randomization by assumption is not a satisfactory way of treating the empirical fact that random contracts are not observed. First, by prohibiting randomization from entering through the front door, one might find it entering in disguised form through the back door. For example, suppose that our moral-hazard model were enriched to allow for several symmetric types of accidents. If random insurance contracts were excluded by assumption, then in some circumstances in which ex ante randomization

In this context it should be noted that the literature on the randomization of insurance contracts, including this article, has not attempted to measure the benefits from randomization, though doing so would not be excessively difficult.

${ }^{21}$ The problem of side-contracts is a pervasive, though neglected, one in contract theory. For instance, whenever payment schedules are nonlinear, there is an incentive for side-payments and the creation of secondary markets. If the payment function is concave, individuals have an incentive to smooth measured output (selling output on the secondary market when output is high, and buying when it is low) and to make measured output more uneven when the payment schedule is convex (e.g., by introducing randomization). The presence of such secondary markets provides one explanation for the linearity of payment schedules.

${ }^{22}$ By treating a monopoly insurer, the analysis assumed that individuals' total purchases of accident insurance are observable. In these circumstances, competitive equilibrium is characterized by exclusivity-each insurance company will require that its clients purchase insurance only from itself. In this case it is not inconsistent to assume away randomization insurance, since accident insurance companies may be able to observe their clients' purchases of randomization insurance as well. Exclusive accident contracts are also enforceable if the accident insurance companies cannot observe individuals' total purchases of accident insurance, but can enforce refusing to make a payment in the event of accident if the insured receives payment from any other company for the accident. This requires that accident insurance companies monitor only victims. With this more limited form of observability, it is not inconsistent to assume that accident insurance firms can enforce exclusive accident insurance contracts, but cannot monitor their clients' purchases of randomization insurance. 
is desirable, competitive equilibrium would entail extended exclusivity (each individual would purchase all his insurance from a single firm) and firms' randomly cross subsidizing between types of insurance, e.g., one firm might run a loss on cancer insurance financed by its profits from automobile accident insurance. Doing so would allow firms to randomize indirectly. Second, one cannot be sure that whatever considerations (discussed above) result in random insurance contracts' not being observed do not also have implications for the structure of nonrandom contracts. For example, if complexity is important, the competitive equilibrium contract may be simple, linear, and nonrandom. But if one were simply to exclude random contracts by assumption, without explicitly treating complexity, the solution might indicate that the equilibrium contract would be highly nonlinear and complex. For both these reasons, disallowing random contracts by assumption is likely to generate spurious results.

\section{Appendix A}

Derivation of $\partial^{2} \mathcal{V} / \partial \Pi^{2}$. Define

$$
V(\Pi, \beta, \hat{e}(\Pi, \beta))=(1-p(\hat{e})) u(w-\beta)+p(\hat{e}) u\left(w-d+\frac{\beta(1-p(\hat{e}))-\Pi}{p(\hat{e})}\right)-\hat{e}
$$

and

$$
Z(\Pi, \beta, \hat{e}(\Pi, \beta))=-p^{\prime}(\hat{e})\left[u(w-\beta)-u\left(w-d+\frac{\beta(1-p(\hat{e}))-\Pi}{p(\hat{e})}\right)\right] .
$$

Then

$$
\frac{\partial \mathcal{V}}{\partial \Pi}=\left.\frac{d V}{d \Pi}\right|_{\beta}=V_{\Pi}+V_{e} \frac{\partial \hat{e}}{\partial \Pi},
$$

where subscripts denote partial derivatives. From the individual's effort choice problem

$$
\frac{\partial \hat{e}}{\partial \Pi}=-\frac{Z_{\Pi}}{Z_{e}} .
$$

Combining (A3) and (A4) gives

$$
\frac{\partial \mathcal{V}}{\partial \Pi}=\frac{1}{Z_{e}}\left(V_{\Pi} Z_{e}-V_{e} Z_{\Pi}\right)
$$

Then

$$
\begin{aligned}
\frac{\partial^{2} \mathcal{V}}{\partial \Pi^{2}} & =\left.\frac{d^{2} V}{d \Pi^{2}}\right|_{\beta}=\frac{\partial\left(\left.\frac{d V}{d \Pi}\right|_{\beta}\right)}{\partial \Pi}+\frac{\partial\left(\left.\frac{d V}{d \Pi}\right|_{\beta}\right)}{\partial \hat{e}} \frac{\partial \hat{e}}{\partial \Pi} \\
& =\left[\frac{1}{Z_{e}^{3}}\left\{Z_{e}\left(V_{\Pi \Pi} Z_{e}^{2}-2 V_{e \Pi} Z_{e} Z_{\Pi}+V_{e e} Z_{\Pi}^{2}\right)-V_{e}\left(Z_{\Pi \Pi} Z_{e}^{2}-2 Z_{e \Pi} Z_{e} Z_{\Pi}+Z_{e e} Z_{\Pi}^{2}\right)\right\}\right] .
\end{aligned}
$$

\section{Appendix B}

- Derivation of $d^{2} E U^{H} / d\left(\alpha_{1}^{L}\right)^{2}$ at the deterministic equilibrium. Let

$$
\begin{array}{ll}
\chi^{k}=\frac{d}{d y}\left(u^{k}\left(w-d+\alpha_{2}^{L}\right)\right) & y^{k}=\frac{d}{d y}\left(u^{k}\left(w-\beta_{2}^{L}\right)\right) \\
X^{k}=\frac{d}{d y}\left(u^{k}\left(w-d+\alpha_{1}^{L}\right)\right) & Y^{k}=\frac{d}{d y}\left(u^{k}\left(w-\beta_{1}^{L}\right)\right) .
\end{array}
$$

Total differentiation of (19) and (20) gives

$$
\begin{array}{ll}
\frac{d \beta_{1}^{L}}{d \alpha_{1}^{L}}=\left(\frac{p^{L}}{1-p^{L}}\right)\left(\frac{X^{L}}{Y^{L}}\right) & \frac{d \alpha_{2}^{L}}{d \alpha_{1}^{L}}=-\left(\frac{X^{L}-Y^{L}}{\chi^{L}-Y^{L}}\right)\left(\frac{y^{L}}{Y^{L}}\right) \\
\frac{d \beta_{2}^{L}}{d \alpha_{2}^{L}}=\left(\frac{p^{L}}{1-p^{L}}\right)\left(\frac{X^{L}}{Y^{L}}\right) & \frac{d \beta_{2}^{L}}{d \alpha_{1}^{L}}=\left(\frac{d \beta_{2}^{L}}{d \alpha_{2}^{L}}\right)\left(\frac{d \alpha_{2}^{L}}{d \alpha_{1}^{L}}\right) .
\end{array}
$$


Now, from (21),

$$
\frac{d E U^{H}}{d \alpha_{1}^{L}}=\left(1-p^{H}\right)\left(-Y^{H} \frac{d \beta_{1}^{L}}{d \alpha_{1}^{L}}-y^{H} \frac{d \beta_{2}^{L}}{d \alpha_{1}^{L}}\right)+p^{H}\left(X^{H}+\chi^{H} \frac{d \alpha_{2}^{L}}{d \alpha_{1}^{L}}\right) .
$$

When the amount of randomization is infinitesmal,

$$
\alpha_{1}^{L}=\alpha_{2}^{L}, \quad \beta_{1}^{L}=\beta_{2}^{L}, \quad Y^{H}=y^{H}, \quad X^{H}=\chi^{H}, \quad d \beta_{1}^{L} / d \alpha_{1}^{L}=-d \beta_{2}^{L} / d \alpha_{1}^{L},
$$

and $d \alpha_{2}^{L} / d \alpha_{1}^{L}=-1$. Hence, at the deterministic equilibrium,

$$
\frac{d E U^{H}}{d \alpha_{1}^{L}}=0
$$

To determine the effect of an infinitesimal randomization, we investigate $d^{2} E U^{H} / d\left(\alpha_{1}^{L}\right)^{2}$. Substituting (A9) into (A10), totally differentiating the resulting expression, and using the above relations when the amount of randomization is infinitesimal give (23).

\section{References}

$\rightarrow$ ARNotT, R.J. AND STIGLitz, J.E. "The Basic Analytics of Moral Hazard." Scandinavian Journal of Economics, Vol. 90 (1988a), pp. 383-413.

- AND — . "Equilibrium in Competitive Insurance Markets with Moral Hazard." Mimeo, 1988b.

BALCER, Y. AND SADKA, E. "Horizontal Equity, Income Taxation, and Self-Selection with an Application to Income Tax Credits." Journal of Public Economics, Vol. 19 (1982), pp. 291-310.

Diamond, P. AND StiglitZ, J. "Increases in Risk and Risk Aversion." Journal of Economic Theory, Vol. 8 (1974), pp. 337-360.

Fellingham, J.C., Kwon, Y.K., AND Newman, D.P. "Ex ante Randomization in Agency Models." RAND Journal of Economics, Vol. 15 (1984), pp. 290-301.

GJeSDAL, F. "Information and Incentives: The Agency Information Problem." Review of Economic Studies, Vol. 49 (1982), pp. 373-390.

HOLMSTROM, B. "Moral Hazard and Observability." Bell Journal of Economics, Vol. 10 (1979), pp. 74-91.

LAFFONT, J.-J. AND MASKIN, E. "The Theory of Incentives: An Overview" in W. Hildenbrand, ed., Advances in Economic Theory, Cambridge: Cambridge University Press, 1982.

MACHINA, M. "The Choice under Uncertainty: Problems Solved and Unsolved." Journal of Economic Perspectives, Vol. 1 (1987), pp. 121-154.

RothSCHILD, M. AND STIGLITZ, J.E. “Increasing Risk: A Definition.” Journal of Economic Theory, Vol. 2 (1970), pp. 225-243.

AND - "Equilibrium in Competitive Insurance Markets: An Essay on the Economics of Imperfect Information." Quarterly Journal of Economics, Vol. 90 (1976), pp. 629-649.

STIGLiTZ, J.E. "Self-Selection and Pareto Efficient Taxation." Journal of Public Economics, Vol. 17 (1982a), pp. 213-240.

. "Utilitarianism and Horizontal Equity: The Case for Random Taxation." Journal of Public Economics, Vol. 18 (1982b), pp. 1-33.

WEISS, L. "On the Desirability of Cheating, Incentives, and Randomness in the Optimal Income Tax." Journal of Political Economy, Vol. 84 (1976), pp. 1343-1352. 